\title{
Bragg reflection from periodic helicoidal media with laterally graded refractive index
}

Sergiy Valyukh, Hans Arwin, J ens Birch and Kenneth J ärrendahl

The self-archived postprint version of this journal article is available at Linköping University Institutional Repository (DiVA):

http:/ / urn.kb.se/ resolve?urn=urn:nbn:se:liu:diva- 142990

N.B.: When citing this work, cite the original publication.

Valyukh, S., Arwin, H., Birch, J ., J ärrendahl, K., (2017), Bragg reflection from periodic helicoidal media with laterally graded refractive index, Optical materials (Amsterdam), 72, 334-340.

https:// doi.org/ 10.1016/j.optmat.2017.06.018

Original publication available at:

https:// doi.org/ 10.1016/j.optmat.2017.06.018

Copyright: Elsevier

http:// www.elsevier.com/

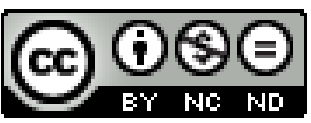




\title{
Bragg reflection from periodic helicoidal media with laterally graded refractive index
}

\author{
S. Valyukh, H. Arwin, J. Birch, K. Järrendahl \\ Department of Physics, Chemistry and Biology, Linköping University, SE-58183 Linköping, Sweden
}

\begin{abstract}
Light interaction with a columnar structure of $\operatorname{In}_{x} A l_{1-x} N$ where each column is a layered periodic helical medium with laterally graded refractive index is considered. It is demonstrated that such a columnar structure can be presented as a stack of layers with a gradient of the refractive index. To calculate reflectance in the proposed model, the $2 \times 2$ characteristic matrix method adopted for a gradient index medium was applied. The influence of the refractive indices (including absorption), parameters of the twisting, and thickness of the periodic structure on reflectance is studied. Cases of normal and oblique incident light are considered. The presented medium is a one-dimensional photonic crystal that can be utilized in many devices for light manipulation.
\end{abstract}

\section{Introduction}

After interaction with a medium, an electromagnetic wave may change its direction of propagation, amplitude and polarization. Development of media for manipulation of electromagnetic waves is of particular interest in fundamental science as well as for practical applications. During the last decades, special attention has been paid to development of novel structured electromagnetic materials with a periodic texture on the scale of the wavelength of the interacting light. When the periodicity of the texture is roughly half the wavelength, these materials scatter photons in a manner similar to scattering of electrons by a crystalline array of atoms in a solid. Such materials possess a photonic bandgap, the optical analogue of the electronic bandgap in semiconductors and are known as photonic crystals [1, 2]. These structures provide opportunities to shape and control the flow of light for optoelectronic and photonic applications [1-3]. Simple examples of one-dimensional (1D) photonic crystals are multilayered periodic structures that have found use in many applications in modern optics for example as filters and reflectors [4,5].

Chiral periodic structures are common in nature [6] and some of them exhibit unique optical properties [7]. For instance, there are no higher diffraction orders at normal incidence, the reflected light is elliptically polarized with the same handedness as the chirality of the structure, the ellipticity depends on the angle of incidence, and the elliptically polarized reflected light does not change its handedness as when it is reflected from achiral media [8]. Also, chiral structures are often employed in various optical systems, e.g. in cholesteric liquid-crystal-based devices [8-10], filters and fiber communication [11]. A feature shared between the natural and artificial chiral periodic structures reported earlier [7-11] is the birefringence of the twisted material, in which the optical axis is rotated in the plane of the structure. However, a twisted periodic medium can be locally isotropic with a helically modulated inhomogeneity. An example of such a medium based on a grading of refractive index is a columnar structure of $\operatorname{In}_{x} \mathrm{Al}_{1-\mathrm{x}} \mathrm{N}$ grown 
on a flat substrate as shown in the scanning electron microscopy image (Fig. 1a) [12]. Each column is a twisted hexagonal structure that can be presented as cylinder with a stack of thin layers, in which the refractive index monotonically changes along a fixed inplane direction within a layer (Fig. 1b). The grading of the refractive index is depicted by shades of gray in Fig. 1b. Black and white correspond to the maximum $\left(n_{\max }\right)$ and minimum $\left(n_{\min }\right)$ values of the refractive index, respectively. The direction of the refractive index variation is uniformly twisted step-wise from one layer to the other.

The $\mathrm{In}_{\mathrm{x}} \mathrm{Al}_{1-\mathrm{x}} \mathrm{N}$ lattice parameter increases internally from the Al-rich side of the column towards the In-rich side, producing a gradient lattice. This lateral gradient in composition and crystalline structure leads to a lateral variation of the physical properties such as band gap, electron mobility and optical constants. Another advantage of the twisted structure of $\operatorname{In}_{x} \mathrm{Al}_{1-\mathrm{x}} \mathrm{N}$ is that the columns are not bent which is the case non-twisted structures of the same material [13]. A regular arrangement of the layers in the column leads to high reflectance in a certain spectral range. The reflectance depends on the structure parameters such as the layer thicknesses, the twist angle between the layers and the period of the structure, and the gradient of the optical constants within the layers. In order to tailor optical properties, it is essential to have an insight into the influence of the structure parameters.

The goal of the present work is to study the reflectance of a periodic helical medium with a laterally graded refractive index as show in Fig. 1b and to explore how the structural parameters affect the optical characteristics. Specifically, the investigation will be carried out for a medium having the optical constants corresponding to $\operatorname{In}_{\mathrm{x}} \mathrm{Al}_{1-\mathrm{x}} \mathrm{N}$ alloys. By doing so, the reflection characteristics of a twisted structure of laterally graded $\operatorname{In}_{x} \mathrm{Al}_{1-\mathrm{x}} \mathrm{N}$ can be predicted and utilized for development of physical devices.

\section{Definition of the problem}

The optical parameters of the columns, in particular the refractive index $n$, and its distribution, depend on the concentration $x$ in the $\operatorname{In}_{x} \mathrm{Al}_{1-\mathrm{x}} \mathrm{N}$ alloy and the sample preparation conditions. This makes it possible to control $n$ and, as a result, the optical properties of the whole structure in a desired way. The refractive index of the $\operatorname{In}_{x} \mathrm{Al}_{1-\mathrm{x}} \mathrm{N}$ alloys has been found [14-16] to have values between $2.1(x=0.1)$ and $2.8(x=0.9)$ in the visible spectral range. The absorption coefficient $\alpha$ can take values between 0 and $2.5 \cdot 10^{5} \mathrm{~cm}^{-1}$ depending on sample preparation conditions. The pitch of the structure and the number of layers per period are defined in the fabrication process.

Although it is evident that the layered periodic structure will cause Bragg reflection, many questions related to details of this reflection arise. For example, it is unclear what the maximum reflectance for unpolarized light will be. Is it 0.5 as for twisted birefringent structures [8] or 1 as for layered achiral media [4]? Intuitively, it is understandable that a higher gradient of the refractive index leads to higher reflection. This is true for small

widths of the columns when the reflection does not reach its saturation. However, without a detailed investigation, it is difficult to draw conclusions about how values of the structural parameters determine the saturation of the reflection. 
The question regarding reflectance of the columnar periodic structure shown in Fig. 1a can be examined by computer simulations. In order to do this, it is necessary to propose a model that will be used for describing the optical properties of the studied structure. In the next section, we will demonstrate that a layered medium gradient index medium in a form of a cylinder (Fig. 1b) can be applied for these purposes. We will, however, allow the cylinder diameter to be much larger that the wavelength of the light making the specular reflection dominate over scattering.

The next step of the study of this model must include a calculation procedure for light interaction. Although this model looks much simpler than the real structure, finding its reflectance is still not a simple optical problem. Analytical and numerical methods for calculation of light propagation in layered isotropic and anisotropic media have been presented in many reports $[4,5,8]$. However, the cylinder shown in Fig. 1b cannot be considered as a medium in which optical properties change only in one direction that is typical for an ordinary layered medium [4]. Because of this, the standard techniques developed for layered one-dimensional (1D) media cannot be applied in the usual way. On the other hand, implementation of the techniques for direct solving Maxwell's equations in three dimensions (3D) is associated with huge computational resources and relatively long computing times [17]. This is a major drawback for an analysis based on numerous outcomes by testing different parameters of the system.

\section{Model}

Consider a thin slice of the columnar structure (Fig. 1a) such that the gradients of the refractive indexes in the columns are orientated in the same direction. Since the columns are cylinders, there is a reason to also take the considered slice in the same form. Let us find an effective medium that affects the light propagation in a similar manner as the considered slice of the columnar structure.

By using the Fresnell-Kirchhoff diffraction formula [4], it is possible to find the angular distribution of the light intensity after its interaction with the considered slice. Assume that we have a normal incidence of a plane wave. For sake of simplicity and due to the symmetry of the problem, it is sufficient to study the integral in the plane of the light incidence and that contains the gradients of the refractive index. In this case, we have

$$
E(\Theta)=C \int_{0}^{L} A(x) \exp \left(-i \frac{2 \pi}{\lambda}(f(x)+x \sin \Theta)\right) d x,
$$

where $\Theta$ is the angle between the normal and the direction of the observation, $C$ is a constant, $L$ is the length of the slice, $A(x)$ and $f(x)$ are the functions described the change of the amplitude and phase of the wave passing the slice, respectively, and $\lambda$ is the wavelength. The identical orientations of the refractive index gradients in the columns cause a deflection of the light. A similar deflection can be achieved by a medium with a gradient of the refractive index. Moreover, this medium can have the same minimum and maximum values of the refractive index as the columns have. It is reasonable to choose such values for further calculation of the Bragg reflection.

By dividing the columnar structure by slices, within which the gradients of the refractive index in the columns are collinear, and replacing these slices with the corresponding effective media, we will obtain a model as shown in Fig. 1b where the 
whole columnar structure is presented as a set of layers. In this case, a cylindrical coordinate system $(\rho, \varphi, z)$ is suitable. The refractive index is a function of all three coordinates

$$
n(\rho, \varphi, z)=0.5\left(n_{\max }+n_{\min }\right)+\frac{\rho}{R}\left(n_{\max }-n_{\min }\right) \cos (\varphi+g(\mathrm{z})) .
$$

Here $n_{\max }$ and $n_{\min }$ are maximum and minimum values of the refractive index, $R$ is the radius of the cylinder, and $g(z)$ is a function described orientation of the refractive index gradient. For an uniform orientation $g(\mathrm{z})=\zeta \mathrm{z}$, where $\zeta$ is a constant.

Although the gradient of $n$ in a real sample does not strictly lie in the $\rho \varphi$-plane, because of inclination of the crystalline grating, for sake of simplicity, we neglect this and assume that $n(\rho, \varphi, z)$ can be presented as a set of sublayers with indices: $n_{z 1}(\rho, \varphi), n_{z 2}(\rho, \varphi) \ldots$ The directions of the gradients of two neighboring layers make a certain angle $\Delta \varphi$ (Fig.1b). The whole structure has the periodicity with the pitch

$$
p=\frac{360^{\circ}}{\Delta \varphi} l
$$

where $l$ is the layers thickness.

In order to find a compromise between $1 \mathrm{D}$ calculations, fast but inadequate in cases of variation of the optical constants in several directions, and rigorous but slow 3D calculations, we applied the following approach. The cylinder presented in Fig. 1b is divided in segments as shown in Fig. 2a. Each segment is considered as an isolated stratified structure with optically uniform layers. The refractive index within a layer is the average value of the refractive index of the area corresponding to this layer in the column

$$
\bar{n}=\frac{1}{S} \int_{S} n(\rho, \phi) \rho d \rho d \phi,
$$

where $S$ is the area of the segment, $\rho$ and $\phi$ are the coordinates in a polar coordinate system and $n(\rho, \phi)$ is the distribution of the complex refractive index within the corresponding segment and layer of the column.

The 2x2 characteristic matrix formalism for solving Maxwell's equations in a stratified medium [4] can be applied for finding the optical response in terms of reflectance $r_{i}$ of the $i^{\text {th }}$ segment. Although each layer in the segment is assumed to be uniform, we make a correction related to the gradient of the refractive index. Let $\chi$ be the angle between the layer normal and the wavevector. In this case the phase shift of the wave passed a layer with the thickness $l$ and the refractive index $n$ is $\delta=\frac{n}{\cos \chi} \frac{2 \pi}{\lambda} l$. If the gradient of the refractive index is parallel to the layers surfaces and lays in the plane of the wave propagation, then the caused phase delay between the place with $n_{\max }$ and $n_{\min }$ is

$$
\Delta \delta=\frac{n_{\max }-n_{\min }}{\cos \chi} \frac{2 \pi}{\lambda} l,
$$

This phase delay leads to the new angle for the light propagation that can be found from the geometrical consideration of the plane wave propagated through the thin layer with 
the graded refractive index (Fig. 2b). If the phase shift between the wave normals of the incident wave at the points $\mathrm{A}$ and $\mathrm{B}$ is $\Lambda$, then this shift between the wave normals of the outgoing wave is $\Lambda+\Delta \delta$. As a result, the outgoing angle can be found as

$$
\sin \chi^{\prime}=\sin \chi+\frac{n_{\max }-n_{\min }}{\cos \chi} \frac{l}{L} .
$$

Therefore, the correction is that we replace the angle $\chi$ in the characteristic matrix of each layer by $\chi^{\prime}$.

Since the areas of the segments depend on the location in the cylinder, the reflectance of the whole cylinder has to be expressed through an area-weighted sum of the segments reflectances $r(\lambda)$

$$
R(\lambda)=\frac{1}{A} \sum_{i=1}^{N} S_{i} r_{i}(\lambda) .
$$

Here $A$ is the total area of the column, $N$ is the number of the segments, and $S_{i}$ is the area of segment $i$.

The reflectances from the cylinder have two contributions: 1) Bragg reflection caused by the periodic structure, and 2) reflection from the top and bottom interfaces between the cylinder and the surrounding media. If the interfaces are flat and the coherence length of the light is sufficiently large, the reflectance caused by the boundaries for a medium with refractive index 2.1 can exceed 0.5 at certain wavelengths due to the interference effect. However, the interfaces of a real sample usually have some surface roughness that suppresses the interference oscillations in the observed spectrum.

In this case the reflectance of the cylinder can be found as an average normalized reflectance of a set of cylinders having flat interfaces but different heights. A variation of the cylinder heights corresponds to a profile of the roughness. A disadvantage of this approach is the increase of the computation time. Practically the same result can be obtained if we use cylinders with flat boundaries but with a coherence length of the light slightly less than the double optical thickness of the cylinder. Another approach for reducing the interference oscillations includes a thick substrate having a refractive index coinciding with the refractive index of the layer closest to the substrate. This approach is the simplest for realization in calculations and was used in this work.

It is assumed that all cylinders are strictly perpendicular to the substrate. In the case when there exists some deviations of the cylinders inclinations, it is possible to consider the system of the cylinders as an ensemble of domains. Each of the domains consists cylinders of the same inclinations. Similar approach was applied for considering non perfectly ordered helicoidal structure in scarab beetles $[18,19]$.

\section{Results and discussion}

By using Eq. (1), we found the angular disturbance of the electromagnetic field passed through a slice of $50 \mathrm{~nm}$ of the columnar structure for the normally incident light. The diameters of the columns in the slice were chosen to be $50 \mathrm{~nm}, n_{\max }=2.5, n_{\min }=2.2$ and the gaps between the columns were $5 \mathrm{~nm} \pm 1 \mathrm{~nm}$. The deflection of the wavevector in such 
geometry is 0.0021 degrees for $\lambda=500 \mathrm{~nm}$. The same deflection is given by a layer of the effective medium having similar thickness, $n_{\max }$, and $n_{\min }$ but with the length $941 \mu \mathrm{m}$. That is, this length can be considered as the diameter of the cylinder shown in Fig. $1 \mathrm{~b}$ and Fig. 2a.

By applying the model described above, the Bragg reflection of the light for different structure parameters was studied. Evidently, the periodicity of the structure has largest impact on the optical response when $p$ is divisible by $\frac{\lambda}{2 n \cos \theta}$, where $\theta$ is the angle of refraction of the light, and $n$ is the average value of the refractive index. For a medium in which the refractive index ranges between $n_{\min }$ and $n_{\max }$ the first order of the Bragg reflection at normal incidence is observed for wavelength $\lambda$ in between $2 p n_{\min } \cos \theta$ and $2 p n_{\max } \cos \theta$. This should be taken into account when choosing the spectral range for calculation of the reflectance. In addition to the wavelength, the reflectance is a function of the twist angle $(\varphi)$, layer thickness $(l)$, cylinder height $(d)$, differences of the refractive indices $\left(n_{\max }-n_{\min }\right)$, extinction coefficient $(k)$ and angle of incidence $(\theta)$. We investigate behavior of spectra of reflectance obtained for different parameters of the structure that are summarized in Table. 1. It is assumed that the incident light is unpolarized. In this case, $r_{i}$ in Eq. (3) is found as the average between two values obtained for two orthogonal polarizations of the light. The investigation related to polarization properties of this structure will be reported in another work. The refractive index of the substrate was taken as $0.5\left(n_{\min }+n_{\max }\right)$. This enables us to reduce interference oscillations related to the multiple reflections from the outer interfaces of the cylinder.

The time of the calculation, as well as the precision, depends on the number of segments $N$. The optimum value of $N$ is mainly governed by the gradient of the refractive index. For the parameters used in our evaluation, the calculation errors in the model are less than a few percent when $N$ is 30 or larger.

The study on influence of the structure parameters on the reflectance is presented in the subsections below. For convenience, we summarize the values of the parameters in each considered case in table 1.

\subsection{Twist angle}

In the cylinder structure, any two neighboring layers characterized by their directions of the refractive index gradient are twisted with respect to each other by a twist angle $\varphi$. This angle defines the number of layers in a period and is related to the pitch and layer thickness by Eq. (1). The calculated spectral reflectances for $\varphi$ equal to $180^{\circ}, 120^{\circ}, 90^{\circ}$ and continuously twisted $(\varphi \rightarrow 0)$ structures are plotted in Fig. 3.

The results in Fig. 3 show that decreasing $\varphi$ leads to an increase of the reflectance maximum. In other words, reflection from a cylinder of thin layers with slightly changing refractive index is higher than reflection from a corresponding cylinder of thick layers. We can also see that the difference between the maximum reflectances for $\varphi=120^{\circ}$, $\varphi=90^{\circ}$ and $\varphi \rightarrow 0^{\circ}$ is not as large as the difference between the maximum reflectances for these values and $\varphi=180^{\circ}$. If the wavelength is far from the range corresponding to the 
Bragg condition, the optical thickness of the cylinder will not be a multiple of $\frac{\lambda}{2}$ and low reflectance is found. In this case, the reflectance of the light from the whole structure is approximately equal to the reflectance from the top interface.

\subsection{Thickness}

Reflectance as a function of the total thickness $d$ or the number of the periods, $N_{p}=\frac{d}{p}$, is shown in Fig. 4, where the majority of the parameters are the same as in the previous calculations (see Table 1). As expected, the reflectance maximum increases with the number of periods. This causes a decrease of the half-height line width. The maximum reflectance versus number of periods can be approximated with

$$
R_{\max }\left(N_{p}\right)=1-A e^{-\frac{N_{p}}{B}},
$$

where $A$ and $B$ are constants. In the case presented in Fig. 4, we find $A=0.85$ and $B=17.6$. It is understandable that other values of $\varphi, p, n_{\min }, n_{\max }, k$, and $\theta$ than the ones presented in Table 1 give other values of $A$ and $B$.

From Fig. 4 we also see that the amplitude and frequency of the spectral oscillations associated with the interference due to multiple reflections from the layers increase with $N_{p}$. The reflectance peak at the large $N_{p}$ exceeds 0.5 - the maximum for a twisted birefringence medium for natural light and it tends to 1 . This means that in contrast to the twisted birefringence medium and like in ordinary stratified medium propagation of both eigen modes are forbidden at the Bragg region. Such a behavior inherent for layered achiral media [4].

\subsection{Differences of the refractive indices}

As mentioned above, the range of Bragg reflection at normal incidence is defined by the products $2 p n_{\min }$ and $2 p n_{\max }$. This predication can be obtained from analysis of the diffraction condition. However, the dependence of the reflectance maximum on the difference between $n_{\max }$ and $n_{\min }$ is complex but it should be expected that a large difference between the refractive indexes should lead to an increase of the Fresnel reflectances at the interfaces. In turn, this should increase the reflectance maximum of the cylinder. In the trivial case when $n_{\max }=n_{\min }$, there is of course no Bragg reflection at all.

To investigate the reflectance change, we have calculated spectral reflectances for different values of $\delta n=n_{\max }-n_{\min }$ as presented in Fig. 5 with parameters as given in Table 1. As seen in Fig. 5, the maximum reflectance as well as the width of the spectral band increase with $\delta n$. As follows from Fig. 5, the reflectance maximum $\left(R_{\max }\right)$ has a steeper increase when comparing small values of $\delta n$. Since $n_{\min }$ is fixed to a value of 2.2 and $n_{\max }$ is varied, we also observe a shift of the reflectance peak wavelength of the first diffraction order according to

$$
\lambda_{\max }=p\left(n_{\max }+n_{\min }\right) .
$$




\subsection{Extinction coefficient}

As already has been indicated, the optical constants of the alloy $\operatorname{In}_{x} A I_{1-x} N$ depend on the conditions during sample preparation [14-16]. This concerns both the real part $n$ and the imaginary part $k$ of the refractive index. In the cases considered earlier, $k=0 \boldsymbol{I n}_{x} \boldsymbol{A} \boldsymbol{I}_{1-\mathrm{x}} \boldsymbol{N}$ is assumed, i.e. there is no absorption in the layer. However, in practice the situation is usually different. So far, we have consider the case when $\mathrm{k}>0$ and the coherence length of the light exceeds the double thickness $d$ of the layer. In other words, the spectrum of the reflected light contains oscillations originating from multiple reflections caused by the planar boundaries of the cylinder. The results for different values of $k$ associated with absorption are plotted in Fig. 6 . The calculations have been done for $\left(n_{\min }, n_{\max }\right)$ varying from 2.3 to $2.5, p=0.11 \mu \mathrm{m}$, the structure is uniformly twisted, normal incidence, and $d=4.4 \mu \mathrm{m}$. (Table 1 ).

As seen in Fig. 6, the maximum value of the Bragg reflectance, as well as the amplitudes of the interference oscillations, decreases as the absorption increases, as expected. For $k=0.05$, there are no interference oscillations and only a weak Bragg reflection is observed, and for $k=0.1$ the reflected light contains mainly the component of the Fresnel reflection from the top interface between the column and air.

\subsection{Angle of incidence}

For oblique incident angles $\theta$ the wavelength $\lambda_{\max }$ of the reflectance maximum of a periodical structure follows from the Bragg condition

$$
\lambda_{\max }=\frac{2 p \sqrt{\left(\frac{n_{\min }+n_{\max }}{2}\right)^{2}-\sin ^{2} \theta}}{m},
$$

where $m$ is the diffraction order. According to Eq. (6), an increase in $\theta$ leads to a decrease in $\lambda_{\max }$, which is known as a blue shift of the interference peak. It is reasonable to expect that $\lambda_{\max }$ of the cylinder under study also satisfies Eq. (6). However, it is difficult to predict the behavior of the maximum value of the reflectance as a function of $\theta$. On the other hand, the reflectance spectral band decreases with $\theta$ and may cause a decrease of the reflectance peak. Which one of these two phenomena that dominates can be understood from the spectra in Fig. 7. It is seen that the reflectance maximum decreases with $\theta$, whereas the blue shift described by Eq. (6) also is observed. Moreover, a white background signal emerges across the spectrum with increasing $\theta$. The background originates mostly due to the Fresnel reflection from the top interface air-cylinder.

\section{Concluding remarks}


In the present work, we simulate light interaction with the columnar structure of $\operatorname{In}_{\mathrm{x}} \mathrm{Al}_{1-\mathrm{x}} \mathrm{N}$ where each column is a layered periodic helical medium having laterally graded refractive index. This structure can be presented as a stack of twisted layers with gradient of the refractive index. To calculate reflectance of this medium, the $2 \times 2$ characteristic matrix adapted for a gradient index matter was applied. The reflectance of such a medium as a function of the structural parameters, in particular the twist angle, thickness, period, refractive indices, and the angle of incidence are studied. The situations when the flat interfaces of the sample causes interference oscillations in the observed spectrum and when the light coherence length is not sufficient to cause such oscillations are considered.

The Bragg reflectance of the studied structure increases when 1) the thickness (number of pitches) increases; 2) the twist angle decreases; 3) the difference between the refractive indices increases; and 4) the absorption decrease. Increasing the angle of incidence leads to a shift of the Bragg reflection peak into the short-wavelength region and a slight decrease of the reflectance peak. In this case, the maximum value of reflectance within the Bragg range decreases slightly whereas the reflectance associated with the Fresnel reflection increases.

Calculations carried out according to the proposed model are much faster and required much less computer recourses in comparison with other numerical methods for solving the Maxwell equations in 3D space. This gives much more freedom in solving inverse and optimization problems on the structure discussed here.

\section{Acknowledgements}

The work has been supported by the Swedish Research Council Formas and The Swedish Energy Agency. 


\section{References}

1. K. Busch, S. Lolkes, R. B. Wehrspohn, H. Foll, Photonic Crystals: Advances in Design, Fabrication, and Characterization, WILEY-VCH Verlag Berlin GmbH, 2003.

2. J.D. Joannopoulos, P.R. Villeneuve, S. Fan, Nature 386 (1997) 143 - 149.

3. J. Villatoro,V. Finazzi,G. Badenes, V. Pruneri, J. Sens. 2009 (2009) 747803,

4. M. Born, E. Wolf, Principles of Optics: Electromagnetic Theory of Propagation, Interference and Diffraction of Light, 7th Ed., Cambridge University Press, 1999.

5. S. Valyukh, I. Valyukh, P. Xu, V. Chigrinov, Jap. J. App. Phys. 45 (2006) 78197826.

6. B. Alberts, A. Johnson, J. Lewis, M. Raff, K. Roberts, and P. Walter. Molecular Biology of the Cell. Taylor and Francis Group, New York, 2002.

7. H. Arwin, R. Magnusson, J. Landin, K. Järrendahl, Phil. Mag. 92 (2012) 15831599

8. V.A. Belyakov, Diffraction Optics of Complex-Structured Periodic Media, Springer, New York, (1992).

9. G. Gilardi, R. Asquini, A. d’Alessandro, G. Assanto, Opt. Exp., 18, (2010) 11524.

10. A. Kozachenko, S. Valyukh, Mol. Crys. Liq. Crys., 367, (2001) 419-426.

11. J. A. Reyes, A. Lakhtakia, Opt. Comm. 259 (2006) 164-173.

12. C.-L. Hsiao, R. Magnusson, J. Palisaitis, P. Sandström, P. O A Persson, S. Valyukh, L. Hultman, K. Järrendahl, J. Birch, Nano lett. 15 (2015) 294-300.

13. G. Z. Radnóczi, T. Seppänen, B. Pécz, L. Hultman, J. Birch, Phys. Stat. Sol. (a) 202 (2005) 76-78.

14. H. He. Y. Cao, R. Fu, H. Wang, J. Huang, C. Huang, M. Wang, Z. Deng, J Mater Sci: Mater. Electron. 21 (2010) 676-681.

15. E. Iliopoulos, A. Adikimenakis, C. Giesen, M. Heuken, A. Georgakilas, App. Phys. Let. 92 (2008) 191907.

16. T. Peng, J. Piprek, G. Qiu, J. O. Olowolafe, K. M. Unruh, C. P. Swann, E. F. Schubert, Appl. Phys. Lett. 71(1997) 2439-2441.

17. W. Yu, X. Yang, Y. Liu, R. Mittra, A. Muto, Advanced FDTD Methods, Arthech House, Boston, 2011.

18. S. Valyukh, H. Arwin, K.Järrendahl, Simulation of Light Scattering from Exoskeletons of Scarab Beetles, Opt. Express 24 (2016) 5794-5808.

19. S. Valyukh, K.Järrendahl, Modeling of light interaction with exoskeletons of scarab beetles, Appl. Opt. 56 (2017) 2510-2516. 
Table 1. Values of the parameters: twist angle $(\varphi)$, thickness $(d)$, refractive indexes $\left(n_{\max }\right.$, $\left.n_{\min }\right)$ and their difference $\left(n_{\max }-n_{\min }\right)$, extinction coefficient $(k)$, incident angle $(\theta)$,and pitch $(p)$ used in the simulations.

\begin{tabular}{|c|c|c|c|c|c|c|c|c|}
\hline Section & $\begin{array}{c}\text { Variable } \\
\text { parameter }\end{array}$ & $\varphi\left(^{\circ}\right)$ & $\begin{array}{c}p \\
(\mu \mathrm{m})\end{array}$ & $\begin{array}{c}d \\
(\mu \mathrm{m})\end{array}$ & $n_{\text {min }}$ & $n_{\max }$ & $k$ & $\theta\left(^{\circ}\right)$ \\
\hline 4.1 & $\varphi$ & $\begin{array}{c}180,120 \\
90, \rightarrow 0\end{array}$ & 0.11 & 2.75 & 2.2 & 2.5 & 0 & 0 \\
\hline 4.2 & $D$ & 90 & 0.11 & $\begin{array}{c}0.55,1.1,1.65 \\
2.2,2.75,3.3, \\
3.85,4.4,8.25\end{array}$ & 2.2 & 2.5 & 0 & 0 \\
\hline 4.3 & $n_{\max }-n_{\min }$ & 90 & 0.11 & 1.1 & 2.2 & $\begin{array}{l}2.3,2.4, \\
2.5,2.6 \text {, } \\
2.7,2.8\end{array}$ & 0 & 0 \\
\hline 4.4 & $K$ & $\rightarrow 0$ & 0.11 & 2.2 & 2.2 & 2.5 & $\begin{array}{c}0,0.01 \\
0.025 \\
0.05 \\
0.1\end{array}$ & 0 \\
\hline 4.5 & $\theta$ & $\rightarrow 0$ & 0.11 & 5.5 & 2.2 & 2.5 & 0 & $\begin{array}{l}0,15 \\
30 \\
45 \\
60,75\end{array}$ \\
\hline
\end{tabular}


a)

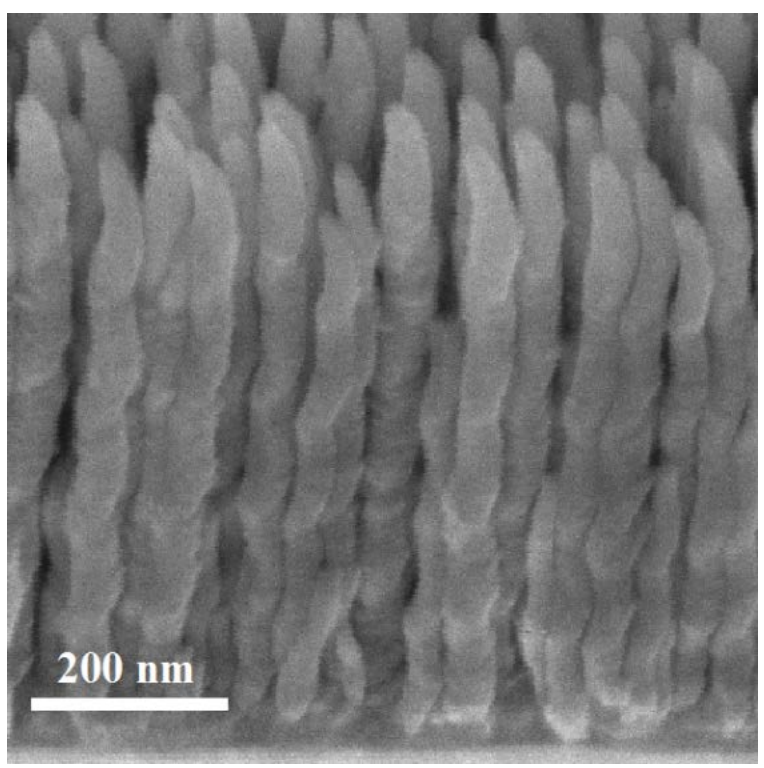

b)

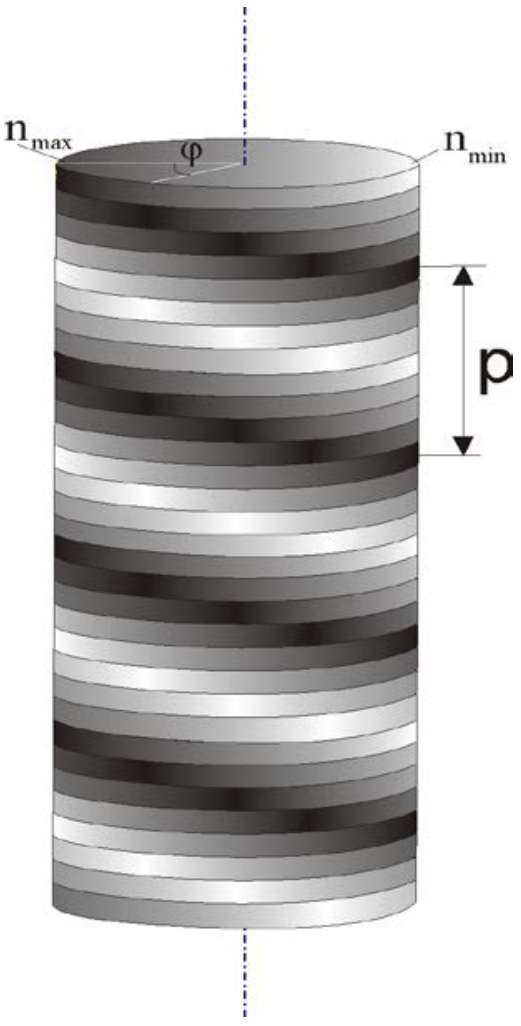

Fig. 1. A chiral periodical medium with laterally graded refractive index. a) A SEM image showing the columnar structure of an $\operatorname{In}_{x} \mathrm{Al}_{1-\mathrm{x}} \mathrm{N}$ alloy, b) model presentation of the $\operatorname{In}_{x} \mathrm{Al}_{1-\mathrm{x}} \mathrm{N}$ alloy in the form of a layered twisted medium with laterally graded refractive index. 
a)

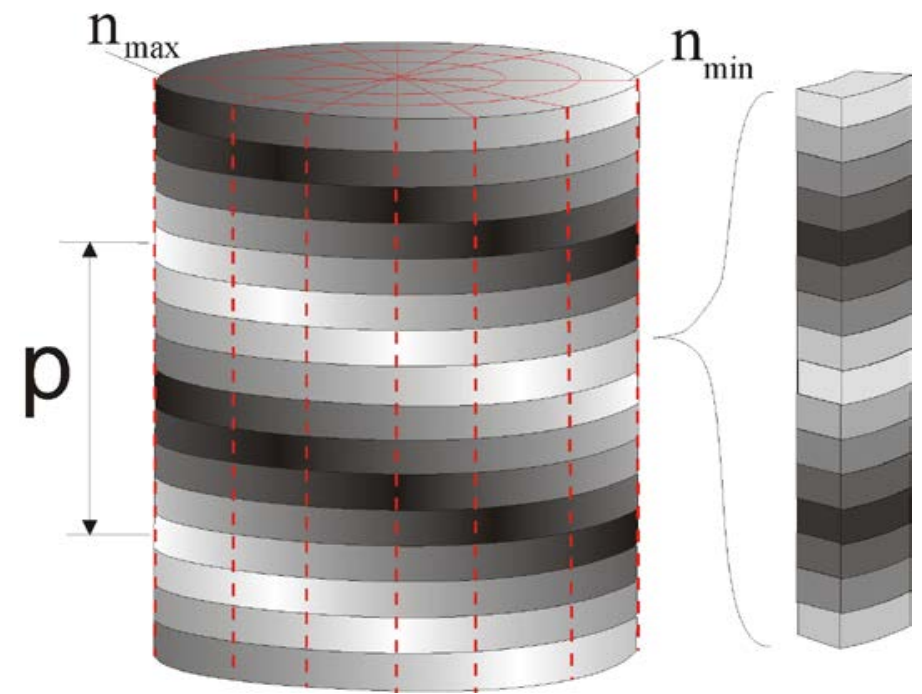

b)

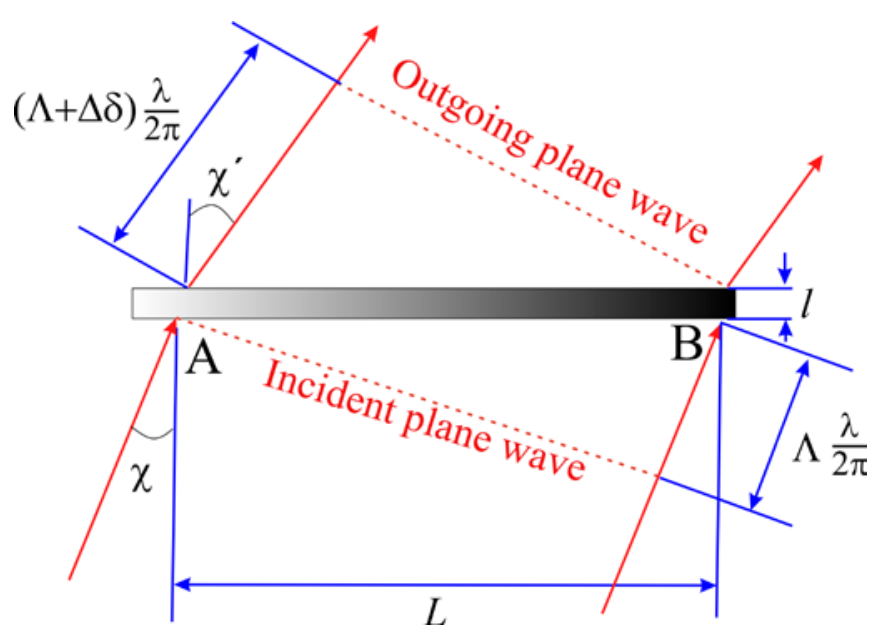

Fig. 2. a) the layered twisted medium is considered as a set of segments, where each segment consists of a stack of uniform layers, $b$ ) the plane wave passed through the layer with to the graded refractive index changes its direction of propagation. 


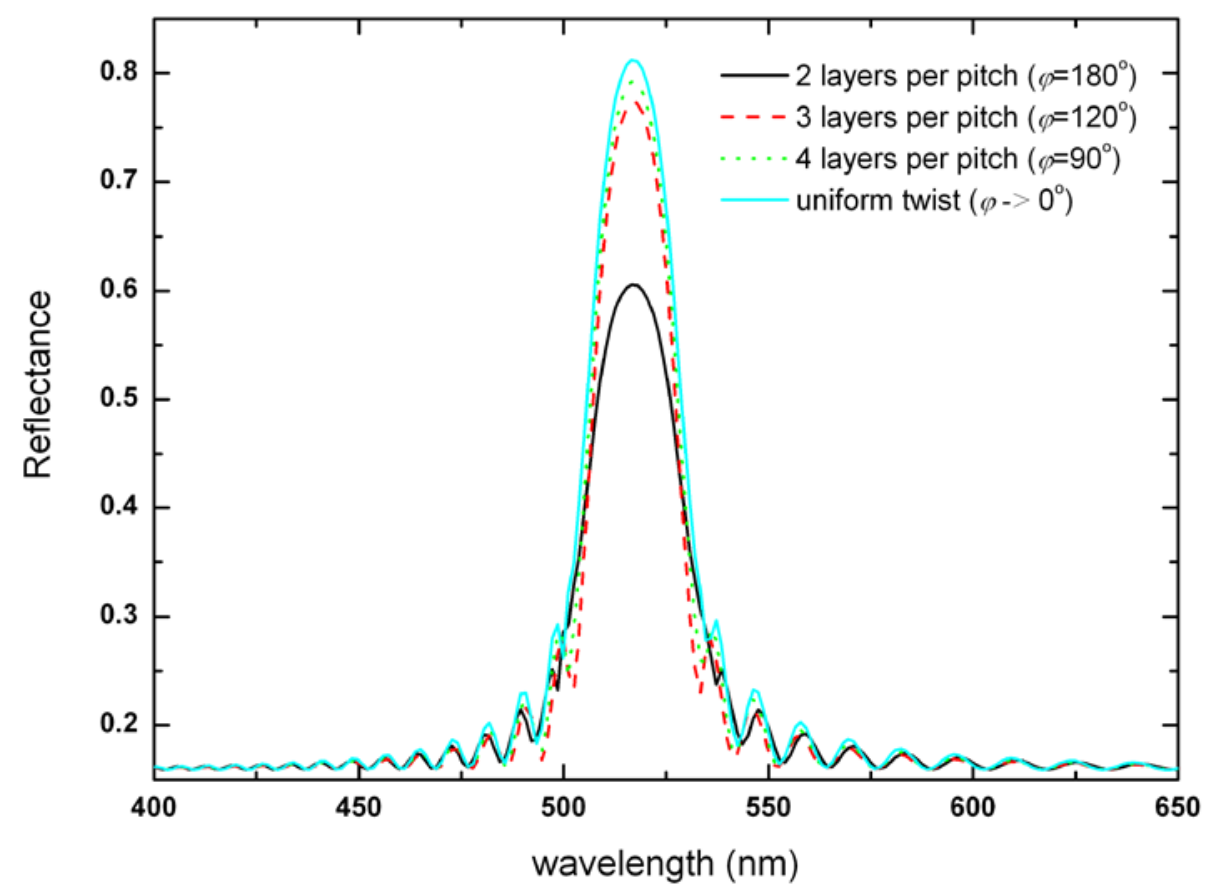

Fig.3. Spectral reflectance for different twist angles $\varphi$ 


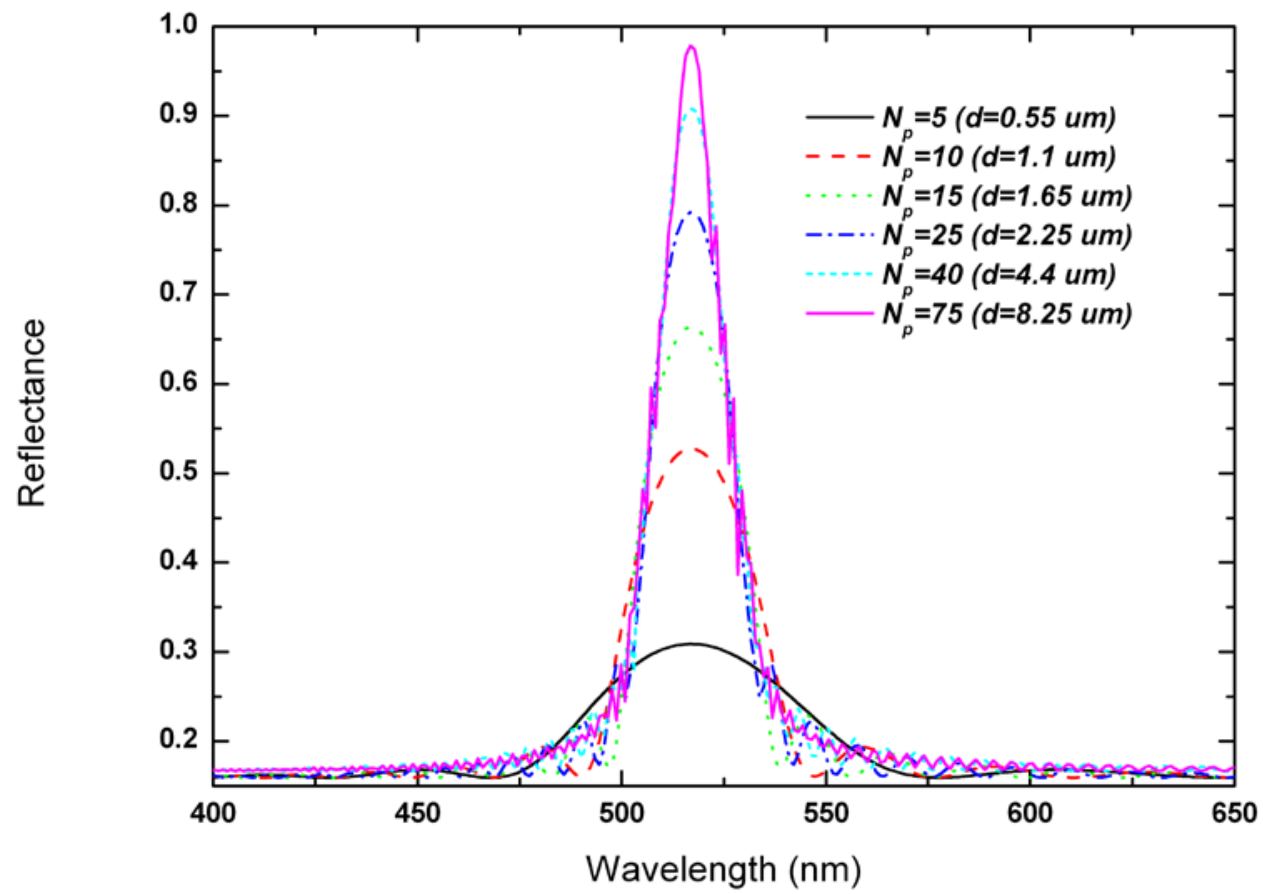

Fig.4. Spectral reflectance for different $N_{p}$ (or thicknesses d). 


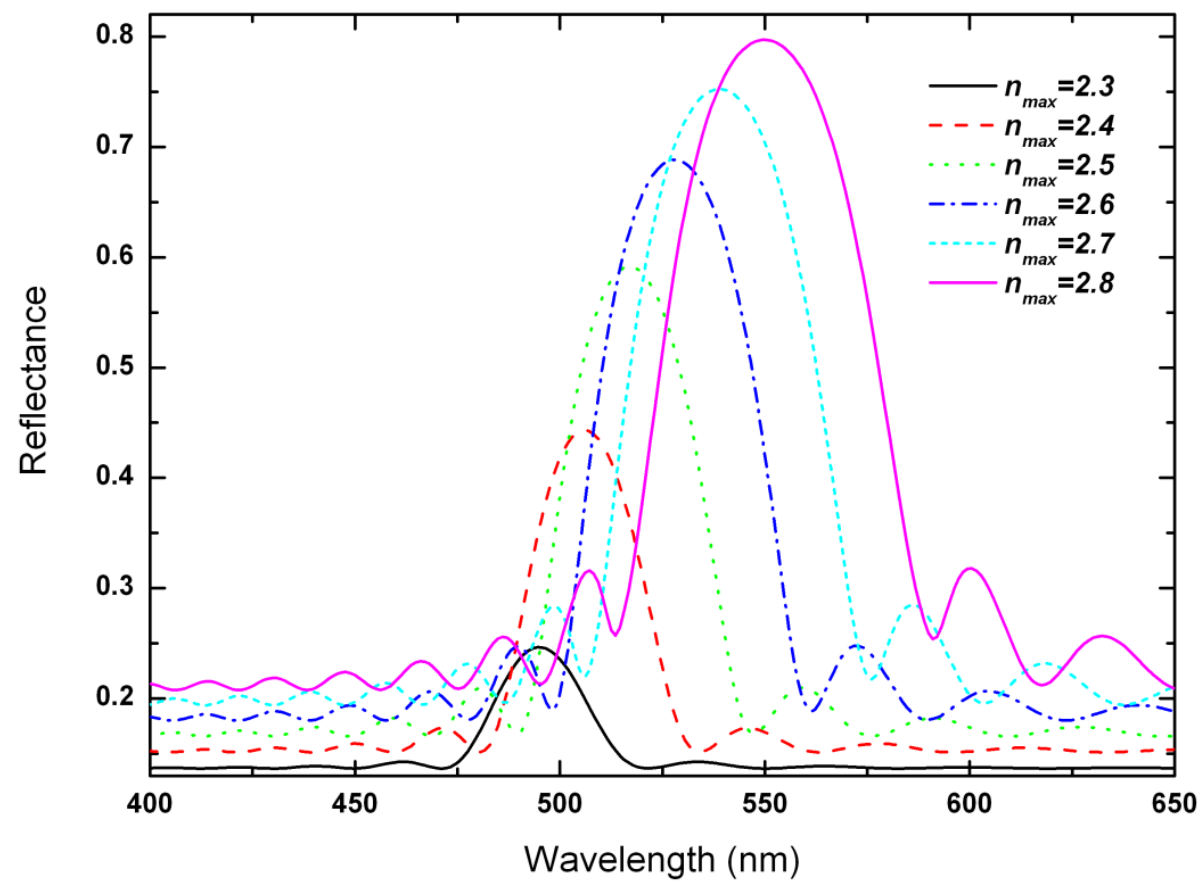

Fig.5. Spectral reflectance for different $\boldsymbol{n}_{\max }$ 


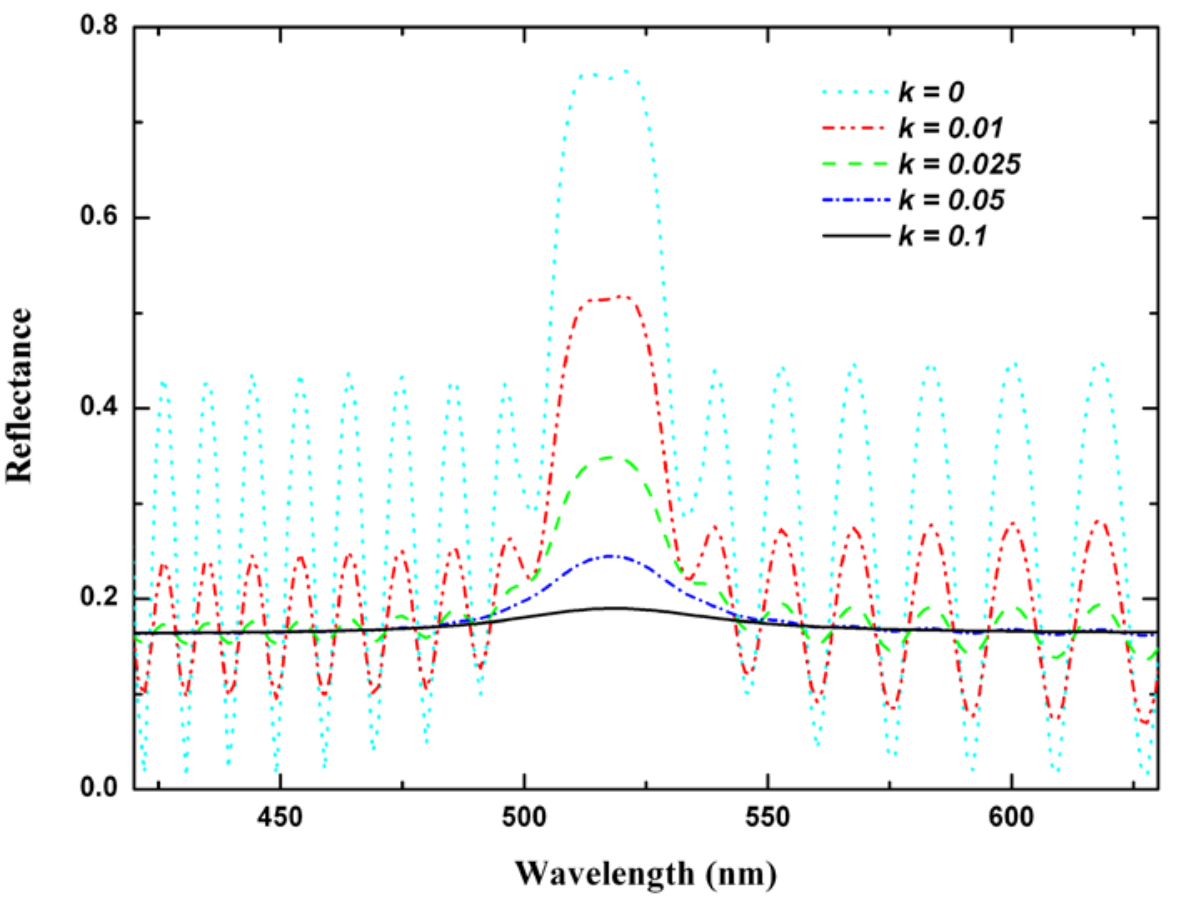

Fig.6. Spectral reflectance for different values of the imaginary part of the refractive index. 


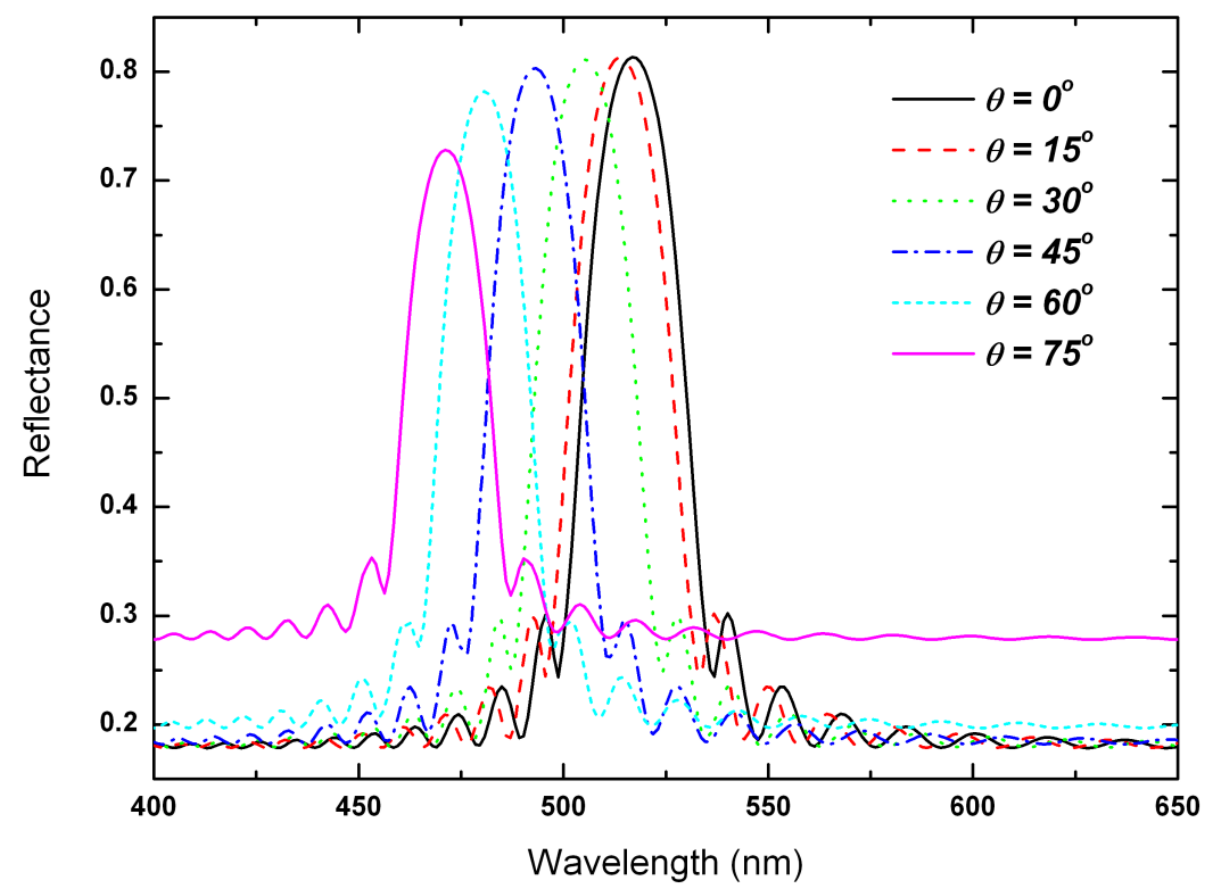

Fig.7. Spectral reflectance for different angles of incidence. 